\title{
Klaus-Michael Köpcke
}

\section{Der Erwerb morphologischer Ausdrucksmittel durch L2-Lerner am Beispiel der Personalflexion}

Der Artikel befaßt sich mit dem Erwerb der Personalfiexion unter natürlichen Erwerbsbedingungen durch erwachsene L2-Lerner. Es wird nachgewiesen, daß sich die Vorstellung cincs geordneten systematischen Verlaufs des natürlichen Zweitspracherwerbs, die sich in der Konzeption von sog. Entwicklungssequenzen niederschlägt, auch für den Erwerb der Personalflexive und dercn Funktion bestätigen läßt.

\section{Einleitung}

In dem vorliegenden Aufsatz soll der Erwerb der Personalflexion unter natürlichen Erwerbsbedingungen durch erwachsene L2-Lerner untersucht werden. Damit wird ein Forschungsgegenstand thematisiert, der in der L2-Forschung bisher nur wenig Beachtung gefunden hat, denn bezüglich des Erwerbs von Flexionsparadigmen bei Verben konzentrierte man sich bisher vor allem auf Tempus- und Aspektmarkierungen, vgl. z. B. Dittmar (1981), Meisel (1986) und Stutterheim (1984). Es ist das Ziel der vorliegenden Arbeit, mittels der exemplarischen Untersuchung eines kleinen Teilbereichs der Grammatik zu einem tieferen Verständnis der menschlichen Sprachlernfähigkeit beizutragen. Zum Begriff der Sprachlernfähigkeit in der L2-Forschung vgl. z. B. Felix (1982) und Wode (1981).

Grundlage für die vorliegende Untersuchung sind die im Rahmen des ZISAProjekts ${ }^{1}$ in den Jahren 1978 bis 1981 erhobenen Longitudinaldaten von 7 Sprechern mit romanischer Muttersprache. Hierbei handelte es sich um vier Italiener, zwei Spanier und eine Portugiesin, die regelmäßig im Abstand von zwei bis vier Wochen von Mitarbeitern des Projekts besucht und interviewt wurden. In der Regel fanden diese Gespräche, die in ihrer Themenstellung von den Interviewern nicht vorstrukturiert waren, in den Wohnungen der L2-Lerner statt, und nicht selten waren andere Familienmitglieder oder Freunde und Bekannte anwesend. Wenn man davon ausgeht, daß erwachsenen L2-Lernern die durch die

1 Hinter dem Akronym ZISA (Zweitspracherwerb italienischer und spanischer Arbeiter) verbirgt sich ein unter der Leitung von Jürgen Meisel (Universităt Hamburg) durchgeführtes Forschungsprojekt zum ungesteuerten Zweitspracherwerb. In dem Projekt wurden eine Querschnittstudie (1977/78) und eine Longitudinalstudie (1978-1982) durchgeführt. Zur Zeit der Auswertung der Longitudinaldaten arbeiteten in dem Projekt außer mir noch Harald Clahsen, Jürgen Meisel, Howard Nicholas und Maryse Vincent mit. Ihnen allen möchte ich an dieser Stelle für Anregungen und geleistete Hilfe danken. 
Kommunikation transportierten Konzepte, nicht jedoch die zu ihrem Ausdruck verwendeten Mittel der Zielsprache bekannt sind, dann wäre es eine wesentliche Aufgabe des Forschers, die Verwendung der Mittel nachzuzeichnen, die zum Ausdruck der verschiedenen Konzepte zu verschiedenen Erwerbszeitpunkten dienen. Longitudinalstudien stellen hierfür die beste Datenbasis bereit.

Die Personalflexive sind im Deutschen aufgrund des bestehenden Kongruenzsystems zwischen Personalflexion und grammatischem Subjekt morphologische Ausdrucksmittel für die Kodierung des Satzsubjekts. Hierbei handelt es sich um eine grammatische Relation, für die im Deutschen auch andere Ausdrucksmittel als die Verbflexive zur Verfügung stehen, z. B. Nominalphrasen oder pronominale Formen. Die vorliegende Arbeit versteht sich in erster Linie als Beitrag zur Erforschung des Erwerbs morphologischer Ausdrucksmittel am Verbkomplex. Ausgangspunkt der Analyse müssen Funktionsbestimmungen sein, nach denen morphologische Ausdrucksmittel klassifiziert werden sollen. Es geht demnach um die Darstellungsabsicht des Lerners. Dies hat zur Konsequenz, daß bei der Analyse der Daten über den Verbkomplex hinausgegangen werden muß. Die Untersuchung des weiteren Redekontexts und die der Kodięung des Satzsubjekts etwa durch lexikalische oder pronominalisierte Subjekte sind erforderlich. Darüber hinaus kann die Funktion der Subjektkodierung auch durch den pragmatischen Bezug auf das in der Gesprächssituation durch den Kommunikationspartner Gesagte hinreichend erfüllt sein. Schließlich kann die grammatische Relation des Subjekts durch Wortstellungsmuster markiert werden, vgl. Clahsen (1984).

Dem funktionalen Ansatz zufolge müssen die formale und die funktionale Ebene der Sprache aufeinander abgebildet werden. Givón (1985: 1018) unterscheidet für den kindlichen Erstspracherwerb zwischen structure (code) und function (message), wobei weiter zwischen dem pragmatischen und syntaktischen Modus der Enkodierung unterschieden wird: "[...] the pragmatic mode is a slow means of processing [...] it is a more transparent communicative system, with a simpler $1: 1$ correlation between code and message. The syntactic mode $[\ldots]$ is a faster, semiautomated mode of processing, but it has lost a certain amount of communicative fidelity and tends to exhibit either a lower correlation between code and message or a more complex correlation."

Zweitsprachenlerner haben in der Regel mehr als ein Mittel zum Ausdruck einer Funktion zur Verfügung; eine Beobachtung, die auch für den kindlichen Erstspracherwerb gilt. Die folgende Übersicht illustriert anhand von LerneräuBerungen die verschiedenen Möglichkeiten zur Markierung des Satzsubjekts. Neben der Angabe des Vornamens des Lerners wird durch Initialen die Muttersprache symbolisiert; die römische Zahl gibt die Nummer des Interviews wieder und die arabische die Dauer des Aufenthalts in Deutschland in Wochen zum Zeitpunkt der Aufnahme. Die Schrägstriche zwischen den Äußerungen der Lerner markieren Äußerungsgrenzen. In Zweifelsfällen wurde für die Ziehung dic- 
scr Grenzen das Kriterium der Intonation herangezogen. Äußerungsteile, die von spitzen Klammern begrenzt werden, wurden von den Lernern getilgt.

Möglichkeiten zur Markierung des Satzsubjekts:

A) Subjckt/Verh-Kongruenz

(1) ich komme von sizile (Bruno, IL; I, 7).

B) Verbmarkierung

(2) 〈du〉 kaufst ein maschine (Giovanni, IL; VII, 25).

C) lexikulische NP

(3) mein bruder for ferie komm in sizile (Bruno, IL; I, 7).

D) Pronomen

(4) er auch gehen naxos (Giovanni, IL; III, 7).

E) situativer Kontext

(5) Interviewer: du hattest mal gesagt, da $B$ du ab januar nicht mehr samstags arbeitest.

Giovanni: ah vielleis / jetz jetz viel arbeiten / und dann arbeiten zehn stunde / und samstag acht uhr an arbeiten (= vielleicht, jetzt arbeite ich viel, 10 Stunden am Tag, und Samstags fange ich um $8 \mathrm{Uhr}$ an zu arbeiten) (Giovanni, IL; VIII, 25).

F) Wortstellung

(6) eine frau italiener freitag komme acht mädchen (= Eine italienische Frau kam Freitag mit acht Mädchen) (Lina, IL; I, 67).

G) Kasusmarkierung

(7) immer trinken der doktor (Bongiovanni, IL; XXI, 51).

\section{Das zielsprachliche System der Verbflexion}

Personen- und Numerusflexive treten im Deutschen als gebundene Morpheme auf. Den sechs semantischen Konzepten der 1., 2. und 3. Pers. Sg./Pl. steht zu deren Ausdruck am Verb nur eine relativ kleine Klasse von Flexiven gegenüber. nämlich $-\emptyset,-e,-(e) s t^{2},-(e) t$ und $-(e) n$. Tabelle I zeigt in Anlehnung an Wurzel

2 Die Einklammerung des $e$ symbolisiert, da $B$ die Realisierung des Schwas in einigen Fällen fakultativ ist. Wurzel (1970) hat gezeigt, daß hierfür phonologische Ursachen gelten. Für die Flexive mit eingeklammerten $e$ bedeutet das, da B das Schwa nicht als Bestandteil der zugrunde liegenden Form betrachtet wird. 
(1970: $63 \mathrm{ff}$.) und Heidolph et al. (1980: $561 \mathrm{ff}$.), welche Flexive zum Ausdruck der verschiedenen grammatischen Personen dienen. Die Darstellung beschränkt sich auf Präsens und Präteritum im Indikativ von Vollverben, da der Modus des Konjunktivs in nahezu allen lernersprachlichen Äußerungen nicht durch Verbflexion markiert wird, sondern stattdessen durch lexikalische Elemente, wie z.B. vielleicht.

\begin{tabular}{|l|l|}
\hline Flexive & grammatische Personen/Tempus \\
\hline$-\emptyset$ & (1.Pers. Sg. Präs.) $)^{3}, 1 . / 3$. Pers. Sg. Prät. \\
$-\mathrm{e}$ & 1.Pers. Sg. Präs. \\
$-(\mathrm{e}) \mathrm{st}$ & 2. Pers. Sg. Präs./Prät. \\
$-(\mathrm{e}) \mathrm{t}$ & 3. Pers. Sg. Präs., 2. Pers. Pl. Präs./Prät. \\
$-(\mathrm{e}) \mathrm{n}$ & 1./3.Pers. Pl. Präs./Prät. . \\
\hline
\end{tabular}

Tab. 1: Distribution der Personalflexive im Deutschen bei Vollverben

Aus Tabelle I ist eine Beschränkung auf das Endparadigma der deutschen Verbflexion ersichtlich. Dieses Vorgehen ist gerechtfertigt, da die Analyse der lernersprachlichen Äußerungen ergeben hat, daß die Flexionsparadigmen für die starken und für die unregelmäßigen Verben, vgl. hierzu Bittner (1985), während des Beobachtungszeitraums entweder gar nicht oder nur als separate Speicherung (sog. rote learning) sprachlicher Einzeltatsachen für einige sehr frequente Verben, wie z. B. sehen (sehe-sie hst - sie ht ), erworben werden. Diese Beobachtủng, die im Deutschen auch im kindlichen Erstspracherwerb festzustellen ist, vgl. Clahsen (im Druck) und Mills (1985), die die Daten von Miller (1976) und Stern/Stern (1907) reanalysiert hat, entspricht im übrigen auch den Überlegungen der Natürlichkeitsgrammatiker, vgl. z. B. Mayerthaler (1981) und Wurzel (1984: 73), die davon ausgehen, daß die ausschließlich additive Beugung der schwachen Verben natürlicher (,normaler") sei als die den Stammvokal modifizierende und zusätzlich additive Beugung der starken Verben. Im übrigen verhalten sich aber auch die starken Verben im Endparadigma völlig analog zu den schwach konjugierten Verben.

Auf eine Darstellung von Ergebnissen zur Entwicklung eines Markierungssystems bei Modalverben und Auxiliaren wurde hier verzichtet, weil für diese beiden Verbtypen entweder die sprachlichen Kontexte fehlen oder sie von einigen Lernern (Ana SL, Giovanni IL und Zita PL) nur im Zusammenhang mit der 1.Pers. Sg. produziert werden.

3 In den deutschen Grammatiken ist die Verwendung des Flexivs $-\emptyset$ im Zusammenhang mit der 1.Pers. Sg. Präs. zwar nicht vorgesehen, jedoch dominiert diese Form zweifelsfrei in der gesprochenen Sprache. $-\emptyset$ wird deshalb von mir als , korrcktc" Markierung der 1. Pers. Sg. Präs. betrachtet. 


\section{Zum Analyseverfahren}

Um den jeweiligen Ausgangspunkt des lernersprachlichen Systems zu erkennen, wurden zunächst vier bis sechs Interviews aus den Anfangsphasen der Erhebungszeiträumc analysiert. Später aufgenommene Interviews wurden in Form von Stichproben untersucht. Wenn sich hierbei gravierende Veränderungen in den lernersprachlichen Systemen abzeichneten, wurden Interviews früher liegender Zeitpunkte herangezogen, bis der jeweilige Beginn der Veränderung erreicht war.

Bei der Analyse der Personen- und Numerusmarkierung im lernersprachlichen System ist insbesondere auf die Verbflexion und im Zusammenhang damit auf den Aufbau eines Kongruenzsystems zu achten. Um überhaupt die lernersprachlichen Systeme ermitteln zu können, müssen die beiden folgenden Voraussetzungen erfüllt sein: 1 . Die vom Lerner verwendeten Formen müssen in verschiedenen, also nicht nur in invarianten Formen auftauchen. Es muß in der Verbverwendung einen paradigmatischen Kontrast geben, wobei die Formen mit bestimmten grammatischen Personen korrespondieren sollten. Schließlich geht es ja darum herauszufinden, ob der Lerner systematische Oppositionen zwischen den zu markierenden grammatischen Personen aufstellt. Dabei ist es wichtig, darauf zu achten, daß das jeweilige vom Lerner verwendete Formeninventar nicht nur mit einem oder zwei Verben verbunden wird. Andernfalls liefe man Gefahr, aus stereotypen Äußerungen oder „formulaic speech“, vgl. WongFillmore (1976), wie ich weiß nich oder verstehst du? systematische Verwendungszusammenhänge abzuleiten. 2 . Die vom Lerner verwendeten Formen sollten systematisch von ihm verwendet werden, nur so lassen sich Form-FunktionsRelationen aufzustellen. Um berechtigterweise von „systematischem Gebrauch" einer Form sprechen zu können, ist es notwendig, ein Mindestkriterium festzusetzen, und zwar: mindestens drei Verwendungen je Form in einem Interview. Werte unterhalb dieser Grenze wären demnach als zufällige Produktionen der Lerner zu betrachten. Das Kriterium ist nicht als Korrektheitsmaß gewählt worden, vgl. Brown (1973), sondern soll lediglich gewährleisten, daß Zufallsproduktionen aus der Analyse ausgeschlossen werden. Andererseits wurde das Mindestkriterium bewußt niedrig angesetzt, da gerade für den L2-Erwerb davon ausgegangen werden kann, daß in vielen Fällen Performanzfaktoren eine schon entwickelte zugrunde liegende Kompetenz, um deren Aufbau in einem grammatischen Teilbereich es hier geht, verdecken können.

Aufgrund dieser Überlegungen wurden die Verbproduktionen eines jeden Lerners in zweifacher Hinsicht analysiert. Zum einen mußte das vom Lerner zu verschiedenen Zeitpunkten verwendete Formeninventar festgestellt werden. Hierzu wurden relative Verwendungshäufigkeiten für die in den sprachlichen Produktionen auftauchenden Verbalaffixe gebildet (= Quotient aus der Verwendungshäufigkeit des Verbalaffixes $X$ und der Summe der Verbproduktionen in Interview Y). Zum anderen mußten die jeweils verwendeten Formen in einen 
funktionalen Zusammenhang zur Markierung der grammatischen Funktion Subjckt gebracht werden, d. h., es mußte geprüft werden, welche Verbalaffixe mit welchen grammatischen Personen zu einem bestimmten Zeitpunkt verwendet werden. Hierzu wurden relative Verwendungshäufigkeiten für die in den Interviews auftretenden Verbalaffixe im Zusammenhang mit den verschiedenen grammatischen Personen gebildet (= Quotient aus der Verwendungshäufigkeit des Verbalaffixes $X$ und der Summe der Verbproduktionen im Zusammenhang mit der grammatischen Person $\mathrm{Y}$ in Interview Z).

\section{Die Ergebnisse der Untersuchung}

\subsection{Der Aufbau eines Formenrepertoires}

Als wesentliches Ergebnis der vorliegenden Untersuchung kann festgehalten werden, daß sich der Erwerb der Markierung von grammatischen Personen und Numerus in einer weitgehend geordneten Reihenfolge vollzieht. Dies gilt in zweierlei Hinsicht, und zwar zum einen, was die Reihenfolge des Auftretens bestimmter Formen am Vollverb betrifft, und zum anderen, was den Ausdruck der verschiedenen grammatischen Personen mittels systematischer Verwendungen von Suffixen am Verb angeht. Für alle Lerner gilt, daß sie die Flexionsendungen nicht in Abhängigkeit von der Verbsemantik gebrauchen, wie dies von Bloom, Lifter und Hafitz (1980) für den monolingualen Erstspracherwerb des Englischen beobachtet wurde. Anstatt also wie im Erstspracherwerb beispielsweise die semantische Unterscheidung zwischen stativen Verben und Handlungsverben auch durch den Gebrauch eines unterschiedlichen Morpheminventars zu berücksichtigen, behandeln die L2-Lerner - wahrscheinlich vor dem Hintergrund ihrer muttersprachlichen Kompetenz - die Kategorie Verb einheitlich.

Alle Lerner verwenden zu Beginn des Zweitspracherwerbs nahezu ausschließlich Verbalsuffixe, Präfixe werden zu keinem Zeitpunkt systematisch zur Markierung von Person und Numerus gebraucht. Das Präfix ge- wird von den Lernern ausschließlich dazu benutzt, temporale und aspektuelle Relationen zu markieren, vgl. Meisel (1986) und Stutterheim (1984). Die von den Lernern verwendeten Suffixe gehören fast ausnahmslos auch zum Formenbestand der Zielvarietät, vgl. Tabelle I. In der Anfangsphase des L2-Erwerbs benutzen fast alle Lerner unabhängig von der zu markierenden grammatischen Person die Verbalsuffixe $-e,-(e) n$ und $-\emptyset$. Für Tabelle II, die das von den Lernern verwendete Formenrepertoire während der Anfangsphase des L2-Erwerbs dokumentieren soll, wurden für jeden Lerner jeweils die drei ersten analysierten Interviews zugrunde gelegt. Ausnahmen stellen Bruno IL und Zita PL dar; da bei diesen Lernern schon ab dem 3 . Interview systematisch die Flexive $-s t$ und $-t$ auftreten, wurden hier jeweils nur die beiden ersten Interviews gewertet. Selbstverständlich produ- 
7.ierten dic Lerner je nach Kommunikationsbereitschaft, Temperament, Stimmung etc. in jedem Interview unterschiedlich viele Verbformen. Für diese erste Auswertung standen für jeden Lerner zwischen 21 (José SL) und 81 (Giovanni IL) Vollverbproduktionen zur Verfügung. Übersicht (8) gibt für jeden Lerner Auskunft über die für Tabelle II zugrunde gelegten Interviews, die Aufenthaltsdaucr und die Summe der Vollverbproduktionen.

Lerner

zugrunde gelegte Intcrviews und Auf- Summe der enthaltsdauer in Wochen

Vollverbproduktion

Ana SL

III, 19; V, 24; VI, 26

63

Bongiovanni IL

I, 7; II, 7; VI, 14

33

Bruno IL

I, 7; II, 11

62

Giovanni IL

I, 3; III, 8; VII, 16

81

José $\mathrm{SL}^{4}$

I, 54; II, 58; III, 63

21

Lina IL

I, 9; II, 12; V, 17

62

Zita PL

III, 19; IV, 21

23

\begin{tabular}{|l|c|c|c|c|c|c|c|}
\hline & $\begin{array}{c}\text { Ana } \\
\text { SL }\end{array}$ & $\begin{array}{c}\text { Bongi. } \\
\text { IL }\end{array}$ & $\begin{array}{c}\text { Bruno } \\
\text { IL }\end{array}$ & $\begin{array}{c}\text { Giov. } \\
\text { IL }\end{array}$ & $\begin{array}{c}\text { José } \\
\text { SL }\end{array}$ & $\begin{array}{c}\text { Lina } \\
\text { IL }\end{array}$ & $\begin{array}{c}\text { Zita } \\
\text { PL }\end{array}$ \\
\hline$-\emptyset$ & .10 & .42 & .45 & .37 & .29 & .19 & .35 \\
$-(\mathrm{e}) \mathrm{n}$ & .41 & .21 & .13 & .48 & .52 & .56 & .26 \\
$-\mathrm{e}$ & .46 & .33 & .39 & .15 & .14 & .23 & .17 \\
Rest* & .03 & .03 & .03 & - & .05 & .02 & .22 \\
\hline Summe & 100 & 99 & 100 & 100 & 100 & 100 & 100 \\
\hline
\end{tabular}

* Die unter der Rubrik „Rest“" aufgeführten Werte beziehen sich auf alle anderen während dieser Phase auftretenden Flexive, also -st und -t oder auf andere von der Zielvarität abweichende Form.

Tab. II: Verwendete Verbalsuffixe während der Anfangsphase des L2-Erwerbs. Angaben in Prozent

4 José SL lebte während seines ersten Aufenthaltsjahres in der BRD weitgehend isoliert von Deutschen, so daß seine Deutschkenntnisse bis zum Zeitpunkt des ersten Interviews durchaus mit denjenigen der anderen Lerner vergleichbar waren. Etwa zum Zeitpunkt des ersten Interviews nahm José SL einen neuen „Job“ als Kellner in einem spanischen Restaurant an; diese Veränderung seiner Lebenssituation ermöglichte ihm endlich den Kontakt zu Deutschen. Vor diesem Hintergrund entwickelte José dann auch Deutschkenntnisse. 
Wenn man bedenkt, daß gerade bei Lernern mit italienischer Muttersprache eine phonologische Interferenz feststellbar ist, Lexeme mit dem Schwa abzuschließen, wie z. B. bei Lina IL in
(9) auch heute komme dottore (VII, 23)

dann relativiert sich der Gebrauch des Flexivs - $c$ durch die italienischen Lerner erheblich, so daß für die Anfangsphase des Erwerbs von Personalflexiven eine dominante Verwendung der Flexive $-\emptyset$ und $-(e) n$ festgestellt werden kann. Die einzige Ausnahme ist Ana SL, die dominant $-e$ und $-(e) n$ verwendet. Auffallig und ebenfalls auf den ersten Blick abweichend von dem Verhalten der übrigen Lerner ist bei Zita PL der in der Rubrik "Rest" eingetragene Wert von $22 \%$. Dieser relativ hohe Wert entsteht jedoch nur, weil zum einen die Gesamtzahl der Vollverbproduktionen während der beiden ersten Interviews mit 23 gering ist und diese Sprecherin zum anderen auch schon in den ersten Interviews in jeweils einem Fall, also deutlich weniger als das Mindestkriterium verlangt, die Flexive -st und $-t$ produziert. Da die festgestellten Flexive sich während der Anfangsphase des L2-Erwerbs bei allen Lernern gleichmäßig über die semantischen Konzepte der 1., 2. und 3. Pers. Sg./Pl. distribuieren, kann geschlußfolgert werden, daß es sich zunächst um unanalysierte Formen handelt, die in keinem funktionalen Zusammenhang zur Markierung des Satzsubjekts am finiten Verb stehen. Die Formenidentität mit der Zielsprache und der in ihr vollzogenen Markierungen der 1. Pers. Sg. und 1./3. Pers. Pl. mit $-\emptyset /-e$ bzw. $-(e) n$ ist also nur zufällig. Bevor jedoch dieser Aspekt des Herstellens eines funktionalen Zusammenhangs zwischen Form und Funktion näher erläutert wird, soll noch kurz die weitere Entwicklung des Formenrepertoires betrachtet werden.

In Tabelle III wurde für jeden Lerner jeweils das erste Interview zugrunde $\therefore$ gelegt, in dem eine neue Form auftaucht, die das oben aufgestellte Mindestkriterium von drei Produktionen erfüllt. Wie schon (8) gibt Übersicht (10) Auskunft über die für Tabelle III zugrunde gelegten Daten.

(10)

Lerner zugrunde gelegte Interviews und Aufenthaltsdauer in Wochen
Summe der

Vollverb-

produktion

Ana SL

$\mathrm{X}, 50$

44

Bongiovanni IL

XXIII, 59

31

Bruno IL

Giovanni IL

III, 13

28

$\mathrm{X}, 31$

34

José SL

VII, 76

41

XVII, 47

23

VI, 31

Zita PL

Jeder Lerner produzierte in diesem Interview zwischen 23 (Lina IL) und 44 (Ana SL) Vollverben. Bei Bongiovanni IL taucht zwar ab dem relativ spät aufge- 
nommenen Interview XXIII mit $-\iota$ cin neues Flexionssuffix auf, jedoch wird diese Form ausschließlich mit den beiden Verben kommen und wiederkommen verknüpft, so da 3 dic oben aufgestellte Forderung, derzufolge es in der Verbverwendung cinen paradigmatischen Kontrast geben muß, nicht erfüllt wird. Vielmehr bildet dieser Lerner im Zusammenhang mit dem Suffix - $t$ lediglich invariante Formen aus. Wenn man von diesen Fällen absicht, und nichts anderes soll dic Einklammerung des Wertes ,..42“ bedeuten, dann gilt für Bongiovanni IL, daß er außer $-\emptyset,-e$ und $-(e) n$ keine weiteren Formen erwirbt. Der gleiche Befund gilt für Lina IL in noch ausgeprägterem Maße, denn bei ihr tauchen auch in dem für Tabelle III zugrunde gelegten späten Interview XVII, aber auch in allen noch später aufgenommenen Interviews nur die schon für die Anfangsphase des Erwerbs festgestellten Formen auf.

\begin{tabular}{|l|c|c|c|c|c|c|c|}
\hline & $\begin{array}{c}\text { Ana } \\
\text { SL }\end{array}$ & $\begin{array}{c}\text { Bongi. } \\
\text { IL }\end{array}$ & $\begin{array}{c}\text { Bruno } \\
\text { IL }\end{array}$ & $\begin{array}{c}\text { Giov. } \\
\text { IL }\end{array}$ & $\begin{array}{c}\text { José } \\
\text { SL }\end{array}$ & $\begin{array}{c}\text { Lina } \\
\text { IL }\end{array}$ & $\begin{array}{c}\text { Zita } \\
\text { PL }\end{array}$ \\
\hline$-\emptyset$ & .02 & .16 & .11 & .24 & .17 & .22 & .23 \\
$-(\mathrm{e}) \mathrm{n}$ & .50 & .32 & .14 & .53 & .37 & .57 & .47 \\
$-\mathrm{e}$ & .34 & .06 & .39 & .06 & .27 & .17 & .10 \\
$-\mathrm{st}$ & - & - & .11 & - & .12 & - & .07 \\
$-\mathrm{t}$ & .14 & $(.42)$ & .25 & .18 & .07 & - & .13 \\
Rest* & - & .03 & - & - & - & .04 & - \\
\hline Summe & 100 & 99 & 100 & 101 & 100 & 100 & 100 \\
\hline
\end{tabular}

* Unter der Rubrik „Rest“ werden in dieser Tabelle solche Verbmarkierungen, die nicht in der Zielsprache auftreten, registriert.

Tab.III: Verwendete Verbalsuffixe zum Zeitpunkt des Auftretens einer neuen Form außer $-e,-(e) n$ und $\emptyset$. Angaben in Prozent

Tabelle III zeigt, daß Bruno IL, José SL und Zita PL die Flexive -st und - $t$ gleichzeitig erwerben, hingegen taucht bei Ana SL und Giovanni IL als neue Form zunächst $-t$ auf. In späteren Interviews produziert Ana SL gelegentlich, jedoch niemals in mindestens drei Fällen je Interview, die Form -st. Das Formenrepertoire von Giovanni expandiert schon kurze Zeit später um -st. Die bis hierhin vorgestellten Ergebnisse bezüglich des Erwerbs eines Formenrepertoires für die Verbflexion lassen sich nun in folgende implikationelle Erwerbsreihenfolge bringen. $\mathrm{Zu}$ der theoretischen Konzeption von Implikationsanalysen vgl. Bailey (1973) und DeCamp (1971).

$$
-s t \supset-t \supset\left\{\begin{array}{l}
-e \\
-\emptyset \\
-(e) n
\end{array}\right\}
$$

Mit anderen Worten: Ein Lerner, zu dessen Repertoire die Form -st zählt, 
wird auch die Form $-t$ produzieren, und ein Lerner, der die Form $-t$ produziert, weist in seinem Formenrepertoire auch die Formen $-e,-\emptyset$ und $-(e) n$ auf. Diese Abfolge gilt jedoch nicht umgekehrt.

Zusammenfassend sind bezüglich der Entwicklung des Formenrepertoires drei Entwicklungsphasen zu unterscheiden:

\begin{tabular}{|c|c|c|}
\hline $\begin{array}{l}\text { (12). } \\
\text { Phase }\end{array}$ & $\begin{array}{l}\text { Kennzeichen } \\
\text { der Phase }\end{array}$ & Zuordnung der Lerner zu den Phasen \\
\hline I & $-\emptyset,-e,-e n$ & $\begin{array}{l}\text { Ana SL, Bongiov. IL, Bruno IL, Giov. IL, } \\
\text { José SL, Lina IL, Zita PL. }\end{array}$ \\
\hline 11 & $-t$ & $\begin{array}{l}\text { Ana SL, Bruno IL, Giovanni IL, José SL, } \\
\text { Zita PL. }\end{array}$ \\
\hline III & - st & $\begin{array}{l}\text { (Ana SL), Bruno IL, Giovanni IL, José } \\
\text { SL, Zita PL. }\end{array}$ \\
\hline
\end{tabular}

Im Mittelpunkt der Untersuchung steht nun aber die Frage: Welche Funktio- . nen drückt das beschriebene Formenrepertoire aus?

\subsection{Das Formenrepertoire und seine Funktion}

Slobin (1973: 184f.) stellt für den monolingualen Erstspracherwerb das Prinzip auf: ,New forms first express old functions, and new functions are first expressed by old forms."

$\mathrm{Zu}$ fragen ist nun, ob dieses Prinzip auch für den ungesteuerten Zweitspracherwerb durch erwachsene Lerner gültig ist. $Z u$ überprüfen wäre demnach, wie das Repertoire semantischer Konzepte, hier also die 1., 2. und 3.Pers.Sg./Pl., von den L2-Lernern aufgebaut wird und weiterhin, ob schon erworbene semantische Konzepte im Verlauf des Erwerbs durch neu erworbene Formen ausgedrückt werden oder ob solche semantischen Konzepte erst dann vom Lerner etabliert werden, wenn die zu ihrem Ausdruck notwendigen Formen zum Repertoire des Lerners zählen.

Im Gegensatz zum kindlichen Erstspracherwerb muß für den ungesteuerten Zweitspracherwerb durch Erwachsene angenommen werden, daß dem Lerner die semantischen Konzepte der 1., 2. und 3.Pers. Sg./PI. von der Muttersprache her bekannt sind. Diese Annahme wird durch die empirischen Befunde der vorliegenden Untersuchung bestätigt, denn alle Lerner realisieren schon in den ersten Interviews die erwähnten semantischen Konzepte. Allerdings geschieht dies mit anderen als in der Zielsprache vorgesehenen Mitteln. Alle Lerner greifen zu frühen Erwerbszeitpunkten darauf zurück, die intendierten Satzsubjekte durch die Wortstellung (vgl. oben Möglichkeit F) zu markieren. Die bevorzugte Wortstellung lautet für alle Lerner SVO, und zwar auch dann, wenn in der Zielsprache eine andere Wortstellung verlangt wird, vgl. Clahsen (1984). Weder 
das Subjekt noch das Objekt des Satzes weisen während dieser Phase Kasusmarkierungen auf. Neben der Möglichkeit, das Satzsubjekt durch kanonische Wortstellungsmuster zu markicren, verlassen sich die Lerner zu Beginn des L2Erwerbs in vielen Fällen aber auch darauf, daß das Satzsubjekt für den Hörer durch den situativen Kontext (Möglichkeit E) erkennbar wird. Schließlich muB darauf hingewiesen werden, daß sich für keinen Lerner eine Korrelation zwischen dem Ausmaß der Subjekttilgungen und dem Erwerb einer neuen Form oder einer eindeutigen Form-Funktions-Relation nachweisen läßt. Darüber hinaus zeigt dic Untersuchung von Nicholas (im Druck), da $B$ es bezüglich der Produktion subjektsloser Sätze zwischen den Lernern ein großes Maß an Variation gibt und daß sich für den Gebrauch solcher Sätze auch dann keine klaren Korrclationen abzeichnen, wenn die Subjekttilgungen in Beziehung zu Entwicklungssequenzen gesetzt werden, die sich über Wortstellungsmuster definieren, vgl. hierzu Clahsen/Meiscl/Picnemann (1983: $97 \mathrm{ff}$.).

Bezüglich des Aufbaus eines Kongruenzsystems kann festgestellt werden, da $B$ während der Anfangsphase des L2-Erwerbs am Verb fast nur die unanalysierten und funktional undifferenzierten Formen $-\emptyset,-e$ und $-(e) n$ auftauchen; ein Kongruenzsystem liegt also noch nicht vor. Das geht auch aus Tabelle IV hervor, für die wieder dieselben Interviews wie schon für Tabelle II zugrunde gelegt wurden. Für Tabelle IV sind sämtliche Kontexte für die verschiedenen grammatischen Personen in den zwei bzw. drei ersten ausgewerteten Interviews zusammengefaßt und dann im Hinblick auf das vom Lerner im Zusammenhang mit der jeweiligen grammatischen Person verwendete Formeninventar hin analysiert worden. Die Einklammerung eines Wertes symbolisiert, daß für das jeweilige Flexiv in sciner Summe über die zwei bis drei Interviews hinweg weniger als drei Anwendungen festgestellt wurden. Dies gilt unter Bezugnahme auf das oben aufgestellte Mindestkriterium. Unter der Rubrik , Rest" sind alle anderen Flexive außer $-\emptyset$, $-e$ und $-(e) n$ oder auch solche Formen, die in der Zielsprache nicht vorkommen, zusammengefaßt worden. In Tabelle IV wird die 2.Pers. Pl. nicht aufgeführt, da diese grammatische Person von allen Lernern fast überhaupt nicht thematisiert wird. Die 1. und 3.Pers. Pl. sind zum einen zusammengefaßt worden, weil sich die Lerner bei diesen beiden grammatischen Personen völlig parallel verhalten haben und zum anderen, weil - zumindest in der Anfangsphase der Erhebung - bei einer getrennten Analyse der 1. und 3. Pers. Pl. nicht in hinreichender Anzahl Kontexte vorgelegen hätten, vgl. das oben erwähnte Mindestkriterium.

Tabelle IV zeigt, daß alle Lerner in der Anfangsphase des L2-Erwerbs die Formen $-\emptyset$, - $e$ und $-(e) n$ über die semantischen Konzepte der 1., 2. und 3.Pers. Sg./Pl. distribuieren. Aufgrund dessen kann darauf geschlossen werden, daß es sich bei $-\emptyset$, $-e$ und $-(e) n$ zunächst noch um Formen ohne grammatische Funktion handelt, die also zunächst noch in keiner Beziehung zur Funktion der Subjektmarkierung stehen. Darüber hinaus ist aus Tabelle IV (und Tabelle II) zu ersehen, daß es zwischen den Lernern bezüglich der Verwendung der Formen - $\theta$, 


\begin{tabular}{|l|c|c|c|c|c|c|c|}
\hline & $\begin{array}{c}\text { Ana } \\
\text { SL }\end{array}$ & $\begin{array}{c}\text { Bongi. } \\
\text { IL }\end{array}$ & $\begin{array}{c}\text { Bruno } \\
\text { IL }\end{array}$ & $\begin{array}{c}\text { Giov. } \\
\text { IL }\end{array}$ & $\begin{array}{c}\text { José } \\
\text { SL }\end{array}$ & $\begin{array}{c}\text { Lina } \\
\text { IL }\end{array}$ & $\begin{array}{c}\text { Zita } \\
\text { PL }\end{array}$ \\
\hline 1. Sg. & $(.07)$ & .40 & .46 & .27 & .40 & .21 & .40 \\
$-\emptyset$ & .68 & .40 & .46 & .21 & $(.07)$ & .21 & $.13)$ \\
$-\mathrm{e}$ & .25 & .16 & $(.08)$ & .52 & .60 & .58 & .33 \\
$-(\mathrm{e}) \mathrm{n}$ & - & .04 & - & - & - & - & - \\
Rest & - & & & & & & \\
\hline 2. Sg. & & $(.67)$ & .70 & .63 & - & .63 & - \\
$-\emptyset$ & - & - & $(.20)$ & - & - & $(.13)$ & - \\
$-\mathrm{e}$ & - & $(.33)$ & $(.10)$ & .37 & - & $(.25)$ & - \\
$-(\mathrm{e}) \mathrm{n}$ & - & - & - & - & $(1)$ & - & $(1)$ \\
Rest & $(1)$ & - & & & & & $(.14)$ \\
\hline 3. Sg. & & & & & & \\
$-\emptyset$ & .17 & $(.40)$ & .31 & $(.11)$ & $(.14)$ & $(.08)$ & $(.33)$ \\
$-\mathrm{e}$ & .35 & $(.20)$ & .38 & $(.11)$ & $(.29)$ & .25 & $(.33)$ \\
$-(\mathrm{e}) \mathrm{n}$ & .43 & $(.40)$ & $(.15)$ & .78 & .57 & .63 & $.17)$ \\
Rest & $(.04)$ & - & $(.15)$ & - & - & $(.04)$ & $(.17)$ \\
\hline $1 . / 3$. Pl. & & & & & & & \\
$-\emptyset$ & - & - & .38 & $(.13)$ & - & - & - \\
$-\mathrm{e}$ & $(.18)$ & - & .38 & .44 & - & $(.29)$ & - \\
$-(\mathrm{e}) \mathrm{n}$ & .82 & - & .23 & .44 & $(1)$ & .71 & - \\
Rest & - & - & - & - & - & - & - \\
\hline
\end{tabular}

Tab.IV: Die verwendeten Formen in Abhängigkeit von ihrer Funktion als Subjektmarkierung bei Vollverben in der Anfangsphase der Erhebung. Angaben in Prozent

\begin{tabular}{llc}
\hline $\begin{array}{l}\text { (13) } \\
\text { Lerner }\end{array}$ & $\begin{array}{l}\text { zugrunde gelegte Interviews und Auf- } \\
\text { enthaltsdauer in Wochen }\end{array}$ & $\begin{array}{l}\text { Summe der } \\
\text { Vollverb- } \\
\text { produktion }\end{array}$ \\
\hline Ana SL & XVII, 76 & 57 \\
Bongiovanni IL & XXXI, 83 & 57 \\
Bruno IL & XVI, 65 & 60 \\
Giovanni IL & XXXII, 94; XXXIV, 110 & 31 \\
José SL & XII, 95 & 25 \\
Lina IL & XXV, 80 & 37 \\
Zita PL & XXV, 107 & 102 \\
\hline
\end{tabular}


$-e$ und $-(c) n$ Variation gibt, denn bei Bongiovanni IL und Bruno IL ist $-\emptyset$ die dominant verwendete Form, bei allen anderen Lernern hingegen - $(e) n$.

$\mathrm{Zu}$ übcrprüfen ist nun, ob das Auftreten einer neuen Form im Lernerrepertoirc auch mit dem Beginn des Aufbaus cines Kongruenzsystems zusammenfallt. Hierzu wurde in Tabelle V das in Tabelle III dargestellte Formenrepertoire der

\begin{tabular}{|c|c|c|c|c|c|c|c|}
\hline & $\begin{array}{c}\text { Ana } \\
\text { SL }\end{array}$ & $\begin{array}{l}\text { Bongi. } \\
\text { IL }\end{array}$ & $\begin{array}{c}\text { Bruno } \\
\text { IL }\end{array}$ & $\begin{array}{c}\text { Giov. } \\
\text { IL }\end{array}$ & $\begin{array}{c}\text { José } \\
\text { SL }\end{array}$ & $\begin{array}{c}\text { Lina } \\
\text { IL }\end{array}$ & $\begin{array}{c}\text { Zita } \\
\text { PL }\end{array}$ \\
\hline $\begin{array}{l}\text { 1.Sg. } \\
-\emptyset \\
-e \\
-(e) n \\
-s t \\
-t \\
\text { Rest }\end{array}$ & $\begin{array}{l}- \\
.70 \\
.30 \\
- \\
- \\
-\end{array}$ & $\begin{array}{c}.23 \\
(.15) \\
.54 \\
- \\
(.08) \\
-\end{array}$ & $\begin{array}{c}.21 \\
.50 \\
.21 \\
- \\
(.07) \\
-\end{array}$ & $\begin{array}{c}(.10) \\
- \\
.90 \\
- \\
- \\
-\end{array}$ & $\begin{array}{l}.20 \\
.32 \\
.48 \\
- \\
- \\
-\end{array}$ & $\begin{array}{c}(.10) \\
.30 \\
.60 \\
- \\
- \\
-\end{array}$ & $\begin{array}{l}.47 \\
.20 \\
.33 \\
- \\
- \\
-\end{array}$ \\
\hline $\begin{array}{l}2 . \mathrm{Sg} . \\
-\emptyset \\
-e \\
-(e) n \\
-s t \\
-t \\
\text { Rest }\end{array}$ & $\begin{array}{l}- \\
- \\
- \\
- \\
-\end{array}$ & $\begin{array}{c}(.17) \\
- \\
(.17) \\
- \\
(.50) \\
(.17)\end{array}$ & $\begin{array}{c}- \\
(.25) \\
- \\
.75 \\
- \\
-\end{array}$ & $\begin{array}{c}.75 \\
- \\
(.25) \\
- \\
- \\
-\end{array}$ & $\begin{array}{c}- \\
- \\
(.28) \\
.71 \\
- \\
-\end{array}$ & $\begin{array}{l}\text { (1) } \\
- \\
- \\
- \\
- \\
-\end{array}$ & $\begin{array}{c}- \\
- \\
(.50) \\
(.50) \\
- \\
-\end{array}$ \\
\hline $\begin{array}{l}3 . \mathrm{Sg} . \\
-\emptyset \\
-\mathrm{e} \\
-(\mathrm{e}) \mathrm{n} \\
-\mathrm{st} \\
-\mathrm{t} \\
\text { Rest }\end{array}$ & $\begin{array}{c}(.08) \\
.25 \\
(.17) \\
- \\
.50 \\
-\end{array}$ & $\begin{array}{c}(.10) \\
- \\
- \\
- \\
(.90) \\
-\end{array}$ & $\begin{array}{l}- \\
.33 \\
- \\
- \\
.67 \\
-\end{array}$ & $\begin{array}{c}(.15) \\
(.08) \\
.31 \\
- \\
.46 \\
-\end{array}$ & $\begin{array}{c}(.20) \\
(.20) \\
.30 \\
- \\
.30 \\
-\end{array}$ & $\begin{array}{c}(.11) \\
- \\
.78 \\
- \\
- \\
(.11)\end{array}$ & $\begin{array}{l}- \\
- \\
.60 \\
- \\
.40 \\
-\end{array}$ \\
\hline $\begin{array}{l}\text { 1./3. Pl. } \\
-\emptyset \\
-\mathrm{e} \\
-(\mathrm{e}) \mathrm{n} \\
-\mathrm{st} \\
-\mathrm{t} \\
\text { Rest }\end{array}$ & $\begin{array}{l}- \\
.22 \\
.78 \\
- \\
- \\
-\end{array}$ & $\begin{array}{l}- \\
- \\
(1) \\
- \\
- \\
-\end{array}$ & $\begin{array}{l}- \\
- \\
(1) \\
- \\
- \\
-\end{array}$ & $\begin{array}{c}(.29) \\
(.14) \\
.57 \\
- \\
- \\
-\end{array}$ & $\begin{array}{l}- \\
(1) \\
- \\
- \\
- \\
-\end{array}$ & $\begin{array}{c}(.67) \\
(.33) \\
- \\
- \\
- \\
-\end{array}$ & $\begin{array}{l}- \\
- \\
(1) \\
- \\
- \\
-\end{array}$ \\
\hline
\end{tabular}

Tab. $V$ : Die verwendeten Formen in Abhängigkeit von ihrer Funktion als Subjektmarkierung bei Vollverben zum Zeitpunkt des Auftretens neuer Formen. Angaben in Prozent 
Lerner zum Zeitpunkt des Auftauchens einer neuen Form in Beziehung zu den semantischen Konzepten der 1., 2. und 3.Pers. Sg./Pl. gesetzt.

Schließlich soll Tabelle VI dokumentieren, wie sich das Verhalten der Lerner bezüglich des Aufbaus eines Kongruenzsystems gegen Ende des Beobachtungs-

\begin{tabular}{|c|c|c|c|c|c|c|c|}
\hline . & $\begin{array}{l}\text { Ana } \\
\text { SL }\end{array}$ & $\begin{array}{l}\text { Bongi. } \\
\text { IL }\end{array}$ & $\begin{array}{c}\text { Bruno } \\
\text { IL }\end{array}$ & $\begin{array}{c}\text { Giov. } \\
\text { IL }\end{array}$ & $\begin{array}{c}\text { José } \\
\text { SL }\end{array}$ & $\begin{array}{c}\text { Lina } \\
\text { IL }\end{array}$ & $\begin{array}{l}\text { Zita } \\
\text { PL }\end{array}$ \\
\hline $\begin{array}{l}1 . \text { Sg. } \\
-\emptyset \\
-e \\
-(e) n \\
-s t \\
-t \\
\text { Rest }\end{array}$ & $\begin{array}{c}.20 \\
.75 \\
(.05) \\
- \\
- \\
-\end{array}$ & $\begin{array}{c}.24 \\
.19 \\
.14 \\
- \\
(.43) \\
-\end{array}$ & $\begin{array}{l}.71 \\
.29 \\
- \\
- \\
- \\
-\end{array}$ & $\begin{array}{l}.77 \\
.23 \\
- \\
- \\
- \\
-\end{array}$ & $\begin{array}{l}.40 \\
.60 \\
- \\
- \\
- \\
-\end{array}$ & $\begin{array}{l}.14 \\
.45 \\
.41 \\
- \\
- \\
-\end{array}$ & $\begin{array}{c}.75 \\
.05 \\
.18 \\
- \\
(.02) \\
-\end{array}$ \\
\hline $\begin{array}{l}\text { 2. Sg. } \\
-\emptyset \\
-e \\
-(e) n \\
-s t \\
-t \\
\text { Rest }\end{array}$ & $\begin{array}{l}- \\
- \\
- \\
- \\
-\end{array}$ & $\begin{array}{c}(.22) \\
- \\
.33 \\
- \\
(.33) \\
(.11)\end{array}$ & $\begin{array}{l}- \\
- \\
- \\
1 \\
- \\
-\end{array}$ & $\begin{array}{l}- \\
- \\
- \\
1 \\
- \\
-\end{array}$ & $\begin{array}{c}(.13) \\
- \\
(.13) \\
.75 \\
- \\
-\end{array}$ & $\begin{array}{l}- \\
- \\
(1) \\
- \\
-\end{array}$ & $\begin{array}{c}- \\
- \\
(.07) \\
.86 \\
(.07) \\
-\end{array}$ \\
\hline $\begin{array}{l}3 . \text { Sg. } \\
-\theta \\
-e \\
-(e) n \\
-s t \\
-t \\
\text { Rest }\end{array}$ & $\begin{array}{c}(.67) \\
- \\
- \\
- \\
(.33) \\
-\end{array}$ & $\begin{array}{c}(.07) \\
.21 \\
.29 \\
- \\
(.42) \\
-\end{array}$ & $\begin{array}{c}- \\
(.08) \\
- \\
(.08) \\
.84 \\
-\end{array}$ & $\begin{array}{c}(.25) \\
- \\
(.25) \\
- \\
.50 \\
-\end{array}$ & $\begin{array}{l}- \\
- \\
- \\
- \\
1 \\
-\end{array}$ & $\begin{array}{l}- \\
.50 \\
.50 \\
- \\
- \\
-\end{array}$ & $\begin{array}{c}(.04) \\
- \\
.50 \\
(.04) \\
.42 \\
-\end{array}$ \\
\hline $\begin{array}{l}\text { 1./3. Pl. } \\
-\emptyset \\
-\mathrm{e} \\
-(\mathrm{e}) \mathrm{n} \\
- \text {-st } \\
-\mathrm{t} \\
\text { Rest }\end{array}$ & $\begin{array}{c}(.08) \\
- \\
.92 \\
- \\
- \\
-\end{array}$ & $\begin{array}{l}- \\
- \\
(1) \\
- \\
- \\
-\end{array}$ & $\begin{array}{l}\bar{z} \\
\overline{1} \\
- \\
\overline{-} \\
-\end{array}$ & $\begin{array}{l}- \\
- \\
- \\
- \\
-\end{array}$ & $\begin{array}{c}- \\
- \\
.89 \\
- \\
(.11) \\
-\end{array}$ & $\begin{array}{c}(.20) \\
(.20) \\
.60 \\
- \\
- \\
-\end{array}$ & $\begin{array}{c}- \\
(.14) \\
.86 \\
- \\
- \\
-\end{array}$ \\
\hline
\end{tabular}

Tab. VI: Die verwendeten Formen in Abhängigkeit von ihrer Funktion als Subjektmarkierung bei Vollverben zum Abschluß des Beobachtungszeitraums. Angaben in Prozent 
zcitraums entwickelt hat. Übersicht (13) zeigt, welche Interviews für Tabelle VI zugrunde gelegt worden sind.

Dic beiden Tabellen V und VI zeigen nun folgendes: Das Suffix $-t$ taucht bei Ana SL ausschließlich im Zusammenhang mit der 3. Pers. Sg. auf. In den später aufgenommenen Intcrviews gibt cs bei dieser Lernerin einzelne Belege für die Anwendung von -st, und zwar immer nur bei der 2. Pers. Sg. Daß diese Belege so sclten sind, liegt an der geringen Anzahl struktureller Kontexte für die 2. Pers. Sg. Ana SL verknüpft also zum Zeitpunkt des Erwerbs einer neuen Form $(-t)$ hiermit auch entsprechend zur Zielsprache das semantische Konzept der 3. Pers. Sg. Da aber bis zum Ende des Erhebungszeitraums weiter die Flexive $-\emptyset$, - $e$ und -en übergeneralisiert werden, vgl. Tabelle VI, kann bei dieser Lernerin nicht von einem vollständigen Erwerb des Kongruenzsystems gesprochen werden.

Bongiovanni IL produziert zwar mit $-t$ eine neue Form, jedoch taucht diese Form nur bei den beiden Verben kommen und wiederkommen auf (genau dies soll durch die Einklammerung der Werte für das Suffix $-t$ in den Tabellen V und VI ausgedrückt werden), zudem wird $-t$ auch noch in den späteren Interviews auf alle semantischen Konzepte übergeneralisiert. Bongiovanni IL erwirbt also bis zum Ende des Erhebungszeitraums weder das zielsprachliche Kongruenzsystem noch Teile daraus, lediglich das Formenrepertoire erfährt bei ihm eine gewisse Erweiterung.

Bruno IL, José SL und Zita PL erwerben die Formen -st und - $t$ gleichzeitig. Während José SL auch in später aufgenommenen Interviews niemals -st übergeneralisiert, finden sich bei Bruno IL und Zita PL vereinzelt Belege für eine Übergeneralisierung von -st auf das semantische Konzept der 3. Pers. Sg. Gelegentliche Übergeneralisierungen von - $t$ gibt es bei Bruno IL im Zusammenhang mit der 1./2.Pers.Sg. und 3.Pers.Pl., bei José SL im Zusammenhang mit der 1.Pers. Sg. und 3.Pers. PI. und bei Zita PL nur im Zusammenhang mit der 1./2. Pers. Sg. Obwohl die drei Lerner die Formen -st und - $t$ zum Zeitpunkt ihres Erwerbs dominant mit den zielsprachlichen Funktionen verknüpfen, kann dies zunächst nicht mit dem vollständigen Erwerb des Kongruenzsystems gleichgesetzt werden, denn vor diesem Schritt, den Bruno IL und José SL in späteren Interviews vollziehen, vgl. Tabelle VI, kann weiterhin die Übergeneralisierung von $-\emptyset$, - $-e$ und $-(e) n$ beobachtet werden. $-\emptyset$ wird von Bruno IL und José SL zunächst noch auf alle semantischen Konzepte übergeneralisiert, von Zita PL hingegen nur auf das semantische Konzept der 3.Pers. Sg.; - $e$ wird von den drei Lernern mit Ausnahme der 2. Pers. Sg. auf alle anderen semantischen Konzepte übergeneralisiert, und -(e) $n$ kann bei Zita PL, wie Tabelle VI zeigt, im Zusammenhang mit allen semantischen Konzepten bis zum Ende des Beobachtungszeitraums festgestellt werden; Bruno IL und José SL nehmen zunächst noch eine Übergeneralisierung dieses Flexivs auf die 1./2. Pers.Sg. respektive 1., 2. und 3.Pers.Sg. vor. Die eindeutige Markierung der semantischen Konzepte der 1./3. Pers. Pl. wird von Bruno IL erst erworben, nachdem er die zielsprachlichen 
(14)

Aufbau des Kongruenzsystems

Lerner

Ana SL

Bruno IL

Giovanni IL

José SL

Zita PL

2. Sg. ว 3. Sg.

1./3. Pl. ว 1. Sg. ว 2./3. Sg.

1./3. PI./1. Sg. ว 2. Sg. ว 3. Sg.

1./3. Pl./1. Sg. ว 2./3. Sg.

1. Sg. ว 2./3. Sg.

Form-Funktions-Relationen für alle grammatischen Personen des Singulars

$\therefore \quad$ (also auch - 0 oder - $e$ für die 1.Pers. Sg.) gelernt hat. Im Unterschied zu Bruno IL erwerben José SL und Zita PL die zielsprachlichen Markierungen für die semantischen Konzepte der 1.Pers. Sg. und 1./3.Pers.Pl. gleichzeitig.

Giovanni IL verknüpft die neu erworbene Form $-t$ ausschließlich mit der 3. Pers. Sg. Auch die später erworbene Form -st wird nur entsprechend zur Zielsprache mit der 2.Pers.Sg. verknüpft. Bevor jedoch das zielsprachliche Kongruenzsystem vollständig erworben wird, nimmt Giovanni IL weiterhin Übergeneralisierungen von $-\phi$ und $-(e) n$ auf alle semantischen Konzepte vor. Wie schon bei Bruno IL, José SL und Zita PL gilt auch für Giovanni IL, daß die zielsprachliche Markierung der semantischen Konzepte der 1./3. Pers. Pl. zuletzt erworben wird. Dem widerspricht nicht, da $B$ Giovanni IL die zielsprachliche Markierung des Konzepts der 1.Pers. Sg. zum gleichen Zeitpunkt wie die für die Konzepte 1./3. Pers. Pl. erwirbt.

Lina IL verwendet während des gesamten Beobachtungszeitraums ohne erkennbare Differenzierung nach semantischen Konzepten ausschließlich die Formen -0 , - $e$ und $-(e) n$, wobei $-(e) n$ durchgängig die dominante Form bleibt.

Unter Vernachlässigung von Bongiovanni IL und Lina IL ergibt sich für die verbleibenden fünf Lerner bezüglich des Erwerbs eines Kongruenzsystems für semantische Konzepte bei Vollverben folgendes Bild, wobei die Ergebnisse wiederum als implikationelle Erwerbsabfolgen dargestellt werden, s. (14).

Aus (14) wird folgendes deutlich:

1. Ana SL und Giovanni IL erwerben die zielsprachliche Markierung für die 3. Pers. Sg. vor der für die 2.Pers.Sg.;

2. der Fall, da B die 2. Pers. Sg. vor der 3. Pers. Sg. erworben wird, tritt nicht auf. Die Markierungen für diese beiden semantischen Konzepte werden böchstens zum gleichen Zeitpunkt erworben, vgl. Bruno IL, José SL und Zita PL;

3. alle Lerner erwerben die Markierungen für die semantischen Konzepte der 2.Pers. Sg. und 3.Pers. Sg. vor derjenigen für die 1.Pers.Sg.; und

4. alle Lerner erwerben die zielsprachliche Kodierung der grammatischen Personen im Plural erst, nachdem eine Differenzjerung für die grammatischen Personen des Singulars abgeschlossen ist. Die Markierung für den Plural wird höchstens zum gleichen Zeitpunkt wie die für die 1.Pers. Sg. erworben.

Somit können als Ergebnis zwei allgemeine Implikationsskalen für den Er- 


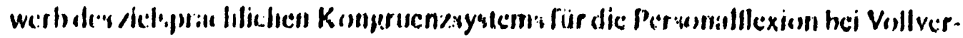
leell illfinglelli werden:

(15) Illuras , Singuluar

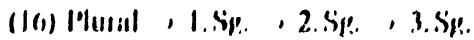

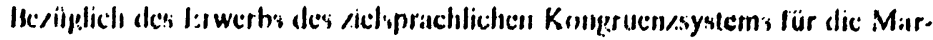

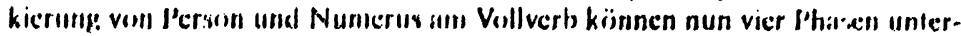
schicken werilen, wic in (17) 1.cecipl.

\begin{tabular}{|c|c|c|}
\hline 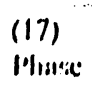 & $\begin{array}{l}\text { Kcoll1/ciclicol } \\
\text { der Ihas:e }\end{array}$ & \%uordinume, der lecrier eu deu lhasen \\
\hline 1 & 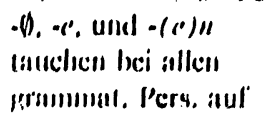 & 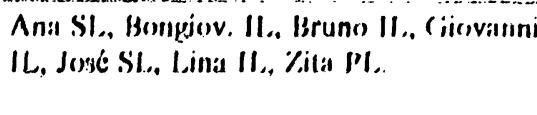 \\
\hline II & $\begin{array}{l}\text {-f Imarkierl dic } \\
\text { 3. I'crs. Sy. }\end{array}$ & $\begin{array}{l}\text { Alla SL, Brumo II. (jiovanni II. José SI., } \\
\text { Lila SL. }\end{array}$ \\
\hline III & $\begin{array}{l}-s / \text { insukierl dic } \\
\text { 2. I'crk. Sg. }\end{array}$ & 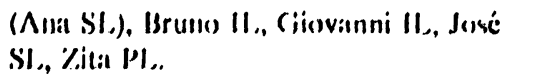 \\
\hline IV & 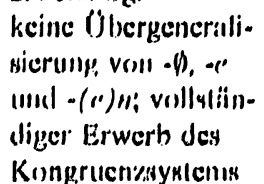 & Brunu IL, Giovauni IL, Jouć SL. \\
\hline
\end{tabular}

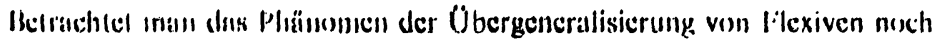

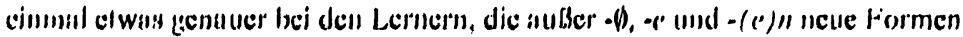

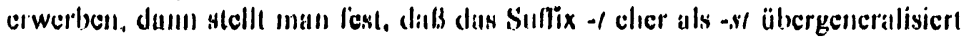
wird. Dies gill nichl nur in (fuantilativer Itinsichl, vgl. hiorzu die Talsellen V und

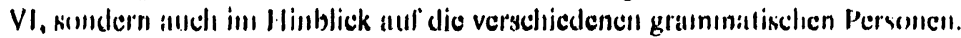

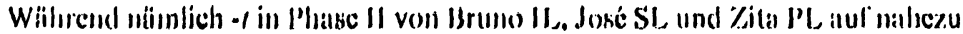

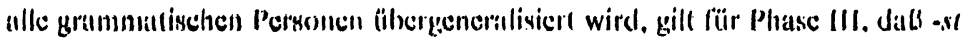
nur von Brino II, und Yita Pl. gelegentlich alul das semantisclic Konzepl der 3. Pers. Sp. ülorgenernlisierl wird. Hingegen wird - / voll diesen beiden Lernern

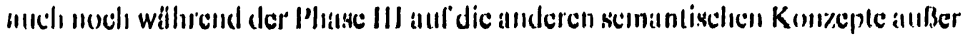

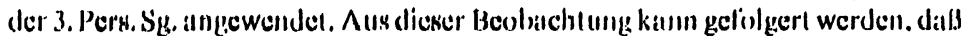

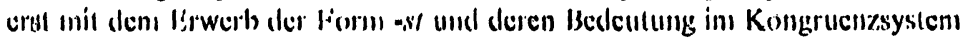
dor Wepg fï̈r dio giclsprachliche Markicrung von l'crson und Numerus gechnet ist. M. II. W.: Wer die liorm -st in ihrer ziclspradiliclion Bedeutung crworhen hal.

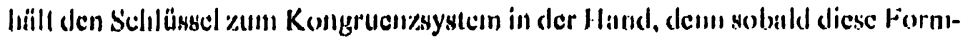

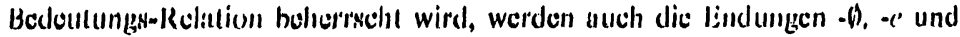

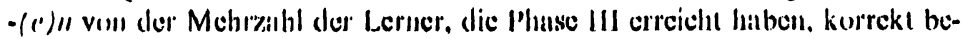
inII\%!. 


\section{Diskussion und Schlußbemerkung}

Alle Lerner bevorzugen zu Beginn des Erwerbs die Formen - $\emptyset$, - $e$ und $-(e) n$. Sie greifen damit - zumindest im Falle von - $\theta$ und $-(c) n$ - genau auf die Formen zurūck, die im Flexionsparadigma der Zielvarietät am häufigsten vorkommen und für die die meisten Anwendungskontexte existieren: bei - $(e)$ n z. B. der Infinitiv, das Partizip Perfekt der sog. starken Verben und die 1./3. Pers. Pl. und bei -0 der Imperativ und die 1 . Pers. Sg. Auch die Form - $t$, die die erste Erweiterung des Formenrepertoires darstellt, ist im Flexionsparadigma der Zielvarietät mehrdeutig. Sie taucht nicht nur als Markierung der 3. Pers. Sg., sondern auch beim Partizip Perfekt der sog. schwachen Verben auf.

Wie die Analyse des Formenrepertoires gezeigt hat, schreiben die Lerner diesen Formen zunächst keine Funktion zu, sondern verwenden sie undifferenziert als unanalysierte Formen. Erst durch die zuletzt erworbene Form -st, die im Unterschied zu den anderen Formen ausschließlich die 2.Pers.Sg. markiert, wird es dem Lerner ermöglicht, die Form-Funktions-Relation für die deutsche Personalflexion zu erschließen. Es ist also vor dem Hintergrund dieser Überlegungen kein Zufall, daß die Lerner zunächst dem semantischen Konzept der 2.Pers. Sg. eine eindeutige Form zuweisen. Eben dies wird auch von Slobin (1973: 203) für den monolingualen Erstspracherwerb vorausgesagt: ,If there are homonymous forms in an inflectional system, those forms will tend not to be the earliest inflections acquired by the child; i.e. the child tends to select phonologically unique forms, when available, as the first realizations of inflections."

Neben dem bisher Gesagten kann zur Erklärung der Ergebnisse aber auch ein auf kommunikativen Funktionen beruhendes Argument entwickelt werden. Im weiteren Sinne der vorliegenden Untersuchung geht es um die Grammatikalisierung von Deixis. Geht man von der kanonischen Äußerungssituation aus, dann impliziert dies, daß einer zu einem oder mehreren anderen spricht, wobei alle Beteiligten einander sehen. Die kanonische Äußerungssituation ist egozentrisch, da der Sprecher und die Rolle des Ego zusammenfallen und die gesamte Situation aus dieser Perspektive ,besprochen“ wird. Es ist natürlich keine Frage, daß die Sprecherrolle in einer Situation wechseln kann. Neben der kommunikativen Rolle des Sprechers (1.Pers.) und der des Adressaten (2. Pers.) ist zu differenzieren nach dem, worüber gesprochen wird, also nach dem Besprochenen (3.Pers.). Von der kanonischen Äußerungssituation geht auch Mayerthaler (1981: 30) in seinen Überlegungen zu morphologischen Natürlichkeit aus. Er argumentiert folgendermaßen: „Ein Sprechakt muß referentiell verankert werden. Da ein Sprechakt [...] Sprecher wie Hörer umfaßt, müssen Sprecher [...] wie Hörer [...] referentiell verankert werden. Um der perzeptiven Deutlichkeit bzw. Transparenz dieser Verankerung willen empfiehlt sich eine merkmalhafte Kodierung der 1. und 2.Person. Symbolisierte man nun die 3.Person gleichermaßen merkmalhaft, dann wäre das resultierende Paradigma nicht ikonisch, da nicht 
asyınmetrisch. Eine Asymmetric kann deshalb nur noch erreicht werden, wenn dic 3. Person (relativ) merkmallos symbolisiert wird [...]."

Mcrkmalhaft wird von den Lernern, wic die Analyse der Daten gezeigt hat, dic 2. Pers. Sg. kodiert; dem stcht cinc merkmallose Kodierung der 1.Pers. Sg. gegenübcr. Gerade hicrdurch werden diese für das Gelingen eines Sprechaktes zentralen Rolle maximal distinktiv markiert. Geht man noch einmal von der cgozentrischen Sprecherperspektive aus, dann scheinen die Lerner über weite Strecken des Erwerbs die Strategie zu verfolgen, die Sprecherrolle, also ihre cigene, unmarkiert (oder zumindest nicht explizit markiert) zu belassen; offensichtlich appellieren die Lerner in frühen Erwerbsphasen des Zweitspracherwerbs an die Kooperationsbereitschaft ihres Kommunikationspartners. Erst wenn diesc Sprecherrolle zugunsten des Adressaten wechseln soli, wird dies in späteren Phasen des natürlichen Zweitspracherwerbs mittels einer expliziten Markierung signalisicrt.

Auf der Grundlage der hier vorgestellten Ergebnisse kann somit als gerechtfertigt festgehalten werden, daß sich die Vorstellung eines geordneten systematischen Verlaufs des natürlichen Zweitspracherwerbs, die sich in der Konzeption der Aufdeckung von Entwicklungssequenzen niederschlägt, vgl. Felix (1982: 73), auch für den Erwerb der Personalflexive und deren Funktion bestätigen läßt.

Zur Erklärung der Befunde wurde einerseits Slobins kognitiver Interpretationsansatz herangezogen. Andererseits und vor allem im Hinblick auf eine funktionale Erklärung der Ergebnisse wurde aber auch auf deiktische Charakteristika einer kanonischen Gesprächssituation verwiesen, die sich auch in verschiedenen Arbeiten zur morphologischen Natürlichkeit wiederfinden.

\section{Literaturnachweis}

[Bailey 1973] Bailey, Charles-James N.: Variation and linguistic theory. - Arlington: Center for Applied Linguistics 1973.

[Bittner 1985] Bittner, Andreas: Wie schwach sind die starken Verben? Überlegungen und Vorschläge zu einer Klassifizierung der neuhochdeutschen Verben. - In: Linguistische Studien. Reihe A. Arbeitsberichte. 126 (Berlin: Akademie der Wissenschaften der DDR, Zentralinstitut für Sprachwissenschaft), 51-74.

[Bloom/Lifter/Hafitz 1980] Bloom, Lois/Lifter, Karin/Hafitz, Jeremie: Semantics of verbs and the development of verb inflection in child language. - In: Language 56 (1980), 386-412.

[Brown 1973] Brown, Roger: A first language: The early stages. - Cambridge, Mass.: Harvard University Press 1973.

[Clahsen 1984] Clahsen, Harald: The acquisition of German word order. A test case for cognitive approaches to second language development. - In: second languages. Ed. by Roger Andersen. - Rowlcy, Mass.: Newbury House 1984. S. 219-242.

[Clahsen 1986] -: Parameterized grammatical theory and language acquisition. A study of the acquisition of verb placement and inflection by children and adults. - In: Linguistic 
Theory and Second Language Acquisition. Ed. by S. Flynn and W. O'Neil. - Dordrecht: Reidel. [Im Druck].

[Clahsen/Meisel/Pienemann 1983] Clahsen, Harald/Meisel, Jürgen/Pienemann. Manfred: Deutsch als Zweitsprache. Der Spracherwerb ausländischer Arbeiter. - Tübingen: Narr 1983.

[DeCamp 1971] DeCamp, David: Implicational scales and sociolinguistic linearity. - In: Linguistics 73 (1971), 30-43.

[Dittmar 1981] Dittmar, Norbert: On the verbal organization of L2-tense marking in an elicited translation task by Spanish immigrants in Germany. - In: Studies in Second Language Acquisition 3 (1981), 136-164.

[Felix 1982] Felix, Sascha: Psycholinguistische Aspekte des Zweitsprachenerwerbs. - Tübingen: Narr 1982.

[Givón 1985] Givón, Talmy: Function, structure, and language acquisition. - In: CrossLinguistic Study of Language Acquisition. Ed. by Dan I. Slobin. - Hillsdale, N. J.: Lawrence Erlbaum 1985. S. 1005-1027.

[Heidolph et al. 1980] Grundzüge einer deutschen Grammatik. Von einem Autorenkollektiv unter der Leitung von Karl Erich Heidolph, Walter Flāmig und Wolfgang Motsch. Berlin: Akademic-Verlag 1980.

[Mayerthaler 1981] Mayerthaler, Wolfgang: Morphologische Natürlichkeit. - Wiesbaden: Athenaion 1981.

[Meisel 1986] Meisel, Jürgen: Reference to past events and actions in the development of natural second acquisition. - In: First and Second Language Acquisition Processes. Ed. by Carol W. Pfaff. - Cambridge, Mass.: Newbury House 1986. S. 206-224.

[Miller 1976] Miller, Max: Zur Logik der frühkindlichen Sprachentwicklung. - Stuttgart: Klett 1976.

[Mills 1985] Mills, Anne: The acquisition of German. - In: The Cross-Linguistic Study of Language Acquisition. Ed. by Dan I. Slobin. - Hillsdale, N. J.: Lawrence Eribaum 1985. S. 141-254.

[Nicholas, im Druck] Nicholas, Howard: Simplifizierung und Auslassungen in der Lernsprache. - In: Sprachentwicklung in der zweiten Sprache. Hrsg. von Maryse Vincent. Tübingen: NarT. [Im Druck].

[Slobin 1973] Slobin, Dan I.: Cognitive prerequisites for the development of grammar. - In: Studies of child language development. Ed. by Charles A. Ferguson and Dan I. Slobin. New York: Holt. Rinehart \& Winston 1973. S. 175-208.

[Stern/Stern 1907] Stern. Clara/Stern, William: Die Kindersprache. - Leipzig. 1907. [Unveränderter reprografischer Nachdruck]: Darmstadt: Wissenschaftliche Buchgesellschaft 1965.

[Stutterheim 1984] Stutterheim, Christiane von: Temporality in learner varieties. A first report. - In: Linguistische Berichte 92 (1984), 31-45.

[Wode 1981] Wode. Henning: Learning a second language: an integrated view of language acquisition. - Tübingen: Narr 1981.

[Wong-Fillmore 1976] Wong-Fillmore, Lilly: The second time around: cognitive and social strategies in second language acquisition. Dissertation Stanford 1976. [Unveröffentlicht].

[Wurzel 1970] Wurzel, Wolfgang Ullrich: Studien zur deutschen Lautstruktur. - Berlin: Akademie-Veriag 1970. = Studia grammatica. 8.

[Wurzel 1984] -: Flexionsmorphologie und Natürlichkeit. Ein Beitrag zur morphologischen Theoriebildung. - Berlin: Akademie-Verlag 1984. = Studia grammatica. 21. 